\title{
Thermal welding of polymeric biomaterials: Development of a novel device for temperature controlled local application
}

Carsten Tautorat, Institute for Biomedical Engineering, Rostock University Medical Center, Rostock-Warnemünde, Germany, carsten.tautorat@uni-rostock.de

Kerstin Lebahn, Institute for Biomedical Engineering, Rostock University Medical Center, Rostock-Warnemünde, Germany, kerstin.lebahn@uni-rostock.de

Stefan Oschatz, Institute for Biomedical Engineering, Rostock University Medical Center, Rostock-Warnemünde, Germany, stefan.oschatz@uni-rostock.de

Daniela Arbeiter, Institute for Biomedical Engineering, Rostock University Medical Center, Rostock-Warnemünde, Germany, daniela.arbeiter@uni-rostock.de

Klaus-Peter Schmitz, Institute for ImplantTechnology and Biomaterials e.V., Rostock-Warnemünde, Germany, klauspeter.schmitz@uni-rostock.de

Niels Grabow, Institute for Biomedical Engineering, Rostock University Medical Center, Rostock-Warnemünde, Germany, niels.grabow@uni-rostock.de

Wolfram Schmidt, Institute for Biomedical Engineering, Rostock University Medical Center, Rostock-Warnemünde, Germany, wolfram.schmidt@uni-rostock.de

\section{Introduction}

Welding techniques play an important role for implant manufacturing. The durability of implant designs highly depends on the quality and reliability of the required welds. To investigate and improve welding techniques, we present our selfdeveloped thermal processing device, enabling local welding of biomaterials, e.g. electrospun nonwovens or polymer films.

\section{Methods}

The modular design of the thermal processing device allows for the usage of application-specific welding tools. The device is controlled by a microprocessor, implemented on our standardized development platform. In this concept study, different geometries of small size heating tips were investigated regarding their suitability for welding of polycarbonate urethane based silicone elastomer (TSPCU) nonwovens. For comparison purposes, the heated surface areas were equal in size $\left(9 \mathrm{~mm}^{2}\right)$. Defined tensile specimens made of TSPCU nonwovens were provided by means of laser cutting. Materials, temperature, heating time and contact pressure were not varied. For estimating suitable heating temperatures we use the differential scanning calorimetry (DSC) to determine thermal material properties. Generated welds were examined by imaging techniques (light microscopy and scanning electron microscopy) and tensile testing.

\section{Results}

The developed welding device allows for heating up to $250{ }^{\circ} \mathrm{C}$. Based on DSC results, we chose a neccessary heating temperature of $90{ }^{\circ} \mathrm{C}$ for our exemplary presented TSPCU nonwovens application, which is in between the melting points of the silicone and polycarbonate urethane blocks of the TSPCU. Thus, a weld can be created, while the polycarbonate urethane backbone remains intact. Regardless of the tip shape, welds were generated withstanding forces of $2 \mathrm{~N}$ and $5 \mathrm{~N}$ for tensile and shear loading, respectively.

\section{Conclusion}

Tensile tests have shown that the thermal processing device is suitable for reproducibly creating firm welds just by heated contact. Depending on the application and choice of materials, we aim to determine appropriate processing parameters for permanent and non-permanent welds. 


\section{Establishment of suitable parameters for laser machining based pro- duction of polymeric implants}

Eric Bohne, Institute for ImplantTechnology and Biomaterials e.V., Rostock-Warnemünde, Germany, e-Mail: eric.bohne@uni-rostock.de

Swen Grossmann, Institute for ImplantTechnology and Biomaterials e.V., Rostock-Warnemünde, Germany, e-Mail: swen.grossmann@uni-rostock.de

Ariane Dierke, Institute for ImplantTechnology and Biomaterials e.V., Rostock-Warnemünde, Germany, e-Mail: ariane.dierke@uni-rostock.de

Stefan Siewert, Institute for ImplantTechnology and Biomaterials e.V., Rostock-Warnemünde, Germany, e-Mail: stefan.siewert@uni-rostock.de

Niels Grabow, Institute for Biomedical Engineering, Rostock University Medical Center, Rostock-Warnemünde, Germany, e-Mail: niels.grabow@uni-rostock.de

Klaus-Peter Schmitz, Institute for ImplantTechnology and Biomaterials e.V., Rostock-Warnemünde, Germany, e-Mail: klaus-peter.schmitz@uni-rostock.de

Michael Stiehm, Institute for ImplantTechnology and Biomaterials e.V., Rostock-Warnemünde, Germany, e-Mail: michael.stiehm@uni-rostock.de

\section{Introduction}

Laser material processing enables precise machining of a wide variety of materials. The complexity of this technology arises from the large number of process-dependent parameters having impact on the cutting results. In order to prevent an excessive heat input, which leads to irreversible material damage, the process parameters have to be adapted to the processed material. To keep the heat load as low as possible, the potential of ultrashort pulse laser technology is exploited. In this regard, we established a workflow and basic parameters for the processing of polymeric material using ultrashort pulse laser technology.

\section{Methods}

As a first step, the required numeric control (NC) file was derived from a given computer aided design (CAD)-design file by means of computer aided manufacturing (CAM)-software. Afterwards, polymeric tubular semi-finished poly-Llactide (PLLA) tubes were placed into the laser system and adjusted orthogonal to the beam. The laser system used is a StarCut Tube system (Coherent, Inc.) with a femtosecond laser and a 4-axis CNC system. The performed parameter studies varying cutting speed, cutting gas pressure and pulse energy to optimize the manufacturing process. Scanning electron microscopy (SEM) was utilized to analyze the cutting results, such as cut edge quality or possible melted areas.

\section{Results}

A parameter set of slow cutting speed, low cutting gas pressure and pulse energy of $25 \mu \mathrm{J}$ was found to be most suitable for PLLA processing. All processed structures could be removed after laser cutting without post-processing. Thus, the material was completely cut through and did not fuse together. The SEM analysis showed that a homogeneous, nonwavy cut edge was created along the kerf. The processed structure did not show any color changes.

\section{Conclusion}

The processing of semi-finished products using femtosecond laser technology is highly depends on the processed material. For this reason, an approach to the laser processing of PLLA is given within this work. The established parameter set is also suitable for processing of very filigree material structures as used in innovative medical devices. 


\section{Comparison of Characteristics of Poly(N-isopropylacrylamide) in Bulk Hydrogel and Ball-milled Microgel Forms}

Jennifer Huling, Institute for Biomedical Engineering, Rostock University Medical Center, Rostock, Germany, e-mail: jennifer.huling@uni-rostock.de

Sabine Illner, Institute for Biomedical Engineering, Rostock University Medical Center, Rostock, Germany, e-mail: sabine.illner@uni-rostock.de

Niels Grabow, Institute for Biomedical Engineering, Rostock University Medical Center, Rostock, Germany, e-mail: niels.grabow@uni-rostock.de

Michael Teske, Institute for Biomedical Engineering, Rostock University Medical Center, Rostock, Germany, e-mail: michael.teske@uni-rostock.de

\section{Introduction}

Poly(N-isopropylacrylamide) (PNIPAM) is one of the most studied temperature sensitive hydrogels and is commonly investigated for biomedical applications due to its proven biocompatibility and a physiologically relevent lower critical solution temperature (LCST). Crosslinked PNIPAM gels are simple to produce and have LCSTs and swelling behaviours which are easy to characterize. Bulk gels can be quickly and easily processed into microgels using a ball mill. However, there is no information on whether we can reliably assume bulk PNIPAM characteristics will be mirrored in microgel particles. Limited initial reports suggest that ball-milling might alter the hydrogel's properties and thermoresponsiveness. Here we compare these key gel characteristics for a series of PNIPAM gels in bulk and ball-milled microgel forms.

\section{Methods}

NIPAM was polymerized with various concentrations of MBAA ( $N, N^{\prime}$-Methylenebisacrylamide) crosslinker to form a series of final hydrogels with variable degrees of swelling and LCSTs. Bulk PNIPAM gel samples were dried completely, then milled into microgel particles using a ball mill. Swelling was accessed by weighing rehydrated bulk hydrogels and microparticles at 25 and $37^{\circ} \mathrm{C}$ in water. LCSTs of bulk gels and particles were measured via differential scanning calorimetry (DSC).

\section{Results}

By varying the concentration of MBAA, bulk PNIPAM hydrogels with swelling ratios ranging from 12.2 to 52.1 were created. The swelling ratios of the microgels were shown to be the same as the bulk materials and all microgels showed the characteristic reduction in swelling above the LCST. The LCSTs, as calculated by DSC, did not significantly differ between bulk gels and microgels.

\section{Conclusion}

PNIPAM can be easily converted into microgels via ball milling. However, it is unknown whether the resulting particles exhibit the same properties as the bulk hydrogels. We have shown that general thermosresponsiveness and the influence of crosslinking on hydrogel swelling were the same in microgels and bulk materials. 


\section{Investigation of dry electromechanical polishing for AM-titanium structures}

Sven Simeunovic, Institute for Medical Engineering and Medical Informatics IM2, School of Life Sciences, University of Applied Sciences Northwestern Switzerland FHNW, 4132 Muttenz, Switzerland, sven.simeunovic@fhnw.ch Christiane Jung, Medical Surface Center, KKS Ultraschall AG, 6442 Steinen, Switzerland, christiane.jung@kks-ultraschall.ch

Dominik Mory, Institute for Medical Engineering and Medical Informatics IM2, School of Life Sciences, University of Applied Sciences Northwestern Switzerland FHNW, 4132 Muttenz, Switzerland, dominik.mory@stud.unibas.ch Daniel Seiler, Institute for Medical Engineering and Medical Informatics IM2, School of Life Sciences, University of Applied Sciences Northwestern Switzerland FHNW, 4132 Muttenz, Switzerland, daniel.seiler@fhnw.ch

Michael de Wild, Institute for Medical Engineering and Medical Informatics IM2, School of Life Sciences, University of Applied Sciences Northwestern Switzerland FHNW, 4132 Muttenz, Switzerland, michael.dewild@fhnw.ch

\section{Introduction}

The post-processing methods of metallic implants has recently been given more attention with the introduction of novel automated polishing methods. One such method is the dry electromechanical polishing process known as DLyte.

The most significant difference to previous electropolishing methods is the use of polymeric sulfonic acid derivative acting as the electrolyte. The characteristic of a solid particle electrolyte raises new questions in terms of polishing results for small features (e.g. tapped holes) as well as overall polishing quality of surfaces.

\section{Methods}

The aim of this study was to determine the quality of the polishing process with titanium rods (supplied by Bibus Metals AG) with tapped holes in three different orientations $\left(0^{\circ}, 45^{\circ}, 90^{\circ}\right)$ as samples by subjecting them to the DLyte dry polishing process for $30 \mathrm{~min}$. In addition, the influence of the voltage and the polarization during the process on the resulting surface quality was determined.

\section{Results}

The increase of the applied voltage from $20 \mathrm{~V}$ to $40 \mathrm{~V}$ led to a lower $\mathrm{R}_{\mathrm{a}}$ value, starting from $0.44 \mu \mathrm{m}$ to $0.23 \mu \mathrm{m}$ for $20 \mathrm{~V}$ whereas for $40 \mathrm{~V}$ the $\mathrm{R}_{\mathrm{a}}$ was lowered from $0.45 \mu \mathrm{m}$ to $0.17 \mu \mathrm{m}$, respectively.

A mechanical polishing beforehand decreased the starting $R_{a}$ value. The afterwards application of the DLyte process led to a further decrease of the $\mathrm{R}_{\mathrm{a}}$ value to $0.12 \mu \mathrm{m}$ for $20 \mathrm{~V}$ and to $0.07 \mu \mathrm{m}$ for $40 \mathrm{~V}$, respectively. An increase of the anodic polarization time from $10 \mu \mathrm{s}$ to $30 \mu \mathrm{s}$ has not led to a significant change in the achievable $\mathrm{R}_{\mathrm{a}}$ values. The data for tapped holes showed no distinct direction dependency for the hole orientation.

\section{Conclusion}

In conclusion, the dry electromechanical polishing process has shown great smoothing capabilities for titanium even with small, tapped holes. The $\mathrm{R}_{\mathrm{a}}$ values were lowered significantly throughout all titanium samples after 30 min polishing time. 


\section{Impact of statins on vascular scaffolds response in porcine carotid arteries}

Sabine Kischkel, Institute for Biomedical Engineering, Rostock University Medical Center, Rostock, Germany, sabine.kischkel@uni-rostock.de

Niels Grabow, Institute for Biomedical Engineering, Rostock University Medical Center, Rostock, Germany, niels.grabow@uni-rostock.de

Carsten M. Bünger, Department of Vascular Medicine, Vivantes Humboldt-Klinikum, Berlin, Germany, carsten.buenger@vivantes.de

Anja Püschel, Department of General, Thoracic, Vascular and Transplantation Surgery, Rostock University Medical Center, Rostock, Germany, anja.pueschel@med.uni-rostock.de

\section{Introduction}

Surgical treatments of arterial occlusive disease with fully absorbable polymeric scaffolds, as a potential alternative to permanent metallic stents, are increasingly penetrating the clinical field. An addition part of the management of patients suffering from vascular diseases is the administration of statins.

\section{Methods}

In this study, absorbable x-ray marked PLLA-based polymer scaffolds and permanent bare-metal stents (BMS) were implanted interventionally into both common carotid arteries (CCA) of 6 healthy female pigs via the left common iliac artery (8F-sheath). The pigs were administered dual antiplatelet drugs oral starting 3 days before the procedure until the end of the study. In Addition, the pigs received atorvastatin orally, beginning 5 days prior to surgery and lasting until the study ended. Stented CCA segments were explanted after 4 weeks, and processed for quantitative histomorphometry, and estimation of vascular inflammation and injury scores.

\section{Results}

Polymer scaffolds showed a decreased residual lumen area and higher stenosis after 4 weeks $\left(6.41 \pm 0.83 \mathrm{~mm}^{2}\right.$ and $40.52 \pm 5.01 \%)$ as compared to the bare-metal reference stent $\left(15.17 \pm 0.896 \mathrm{~mm}^{2}\right.$ and $\left.7.80 \pm 0.88 \%\right)$. After 4 weeks, inflammation score were higher in the polymer group $(1.30 \pm 0.37)$ compared to the BMS group (0.42 \pm 0.18$)$. In contrast, the BMS showed a slightly elevated vascular injury score $(0.85 \pm 0.12)$, as compared to the polymer $(0.60 \pm 0.23)$ group.

\section{Conclusion}

In this preclinical model, the new absorbable polymeric scaffolds showed similar technical feasability and safety for vascular application as the permanent metal stents. Although no positive trends were observed with oral treatment with atorvastatin, further optimization with a dual-loaded coating is still reasonable. In addition, reduced strut thickness of the polymer scaffolds would have potential to positively impact tissue ingrowth between struts and should be considered in future work on stent design. 


\title{
Mechanical properties of different 3D-printed polycaprolactones for ten- don implant applications
}

\author{
Merle Kempfert, Hannover Medical School, Department of Orthopedic Surgery, NIFE, Hannover, Germany, \\ Kempfert.merle@mh-hannover.de \\ Nina Angrisani, Hannover Medical School, Department of Orthopedic Surgery, NIFE, Hannover, Germany, An- \\ grisani.nina@mh-hannover.de \\ Cornelia Blume, Leibniz University Hannover, Institute of Technical Chemistry, Hannover, Germany, blume@iftc.uni- \\ hannover.de \\ Sebastian Loewner, Leibniz University Hannover, Institute of Technical Chemistry, Hannover, Germany, \\ loewner@iftc.uni-hannover.de \\ Yvonne Roger, Hannover Medical School, Department of Orthopedic Surgery, NIFE, Hannover, Germany, Rog- \\ er.yvonne@mh-hannover.de \\ Elmar Willbold, Hannover Medical School, Department of Orthopedic Surgery, NIFE, Hannover, Germany, Will- \\ bold.elmar@mh-hannover.de \\ Janin Reifenrath, Hannover Medical School, Department of Orthopedic Surgery, NIFE, Hannover, Germany, Rei- \\ fenrath.janin@mh-hannover.de
}

\section{Introduction}

Tendon tears are common disorders, which often result in long-term-pain and disfunction. High rerupture rates after surgical refixations implicate that implants should provide sufficient mechanical strengths and cell ingrowth. Polycaprolactone (PCL) is an FDA-approved material, which is available in different molecular weights and different purities. 3D-printed mats from two different PCLs with different designs were produced and tested mechanically.

\section{Methods}

PCL pellets with lower $\left(M_{n} 45000\right.$, Sigma) and higher $\left(M_{n} 97000\right.$, ITV Denkendorf) molecular weights were purchased. Medical grade purity was available only for $M_{n} 97000$ PCL. General printing parameters $\left(80{ }^{\circ} \mathrm{C}, 700 \mathrm{kPa}, 8 \mathrm{~mm} / \mathrm{s}\right)$ were chosen as previously developed using a BioXCellink. Each of the three layers per mat consisted of a meandering pattern with an offset to the next layer of $45^{\circ}$ and $90^{\circ}$, respectively. Test specimens were evaluated with a material testing machine (Zwick 1445). Statistics was performed using SPSS.

\section{Results}

The above chosen printing parameters worked well for PCL $\mathrm{M}_{\mathrm{n}} 45000$, but had to be adapted for the $\mathrm{M}_{\mathrm{n}} 97000$ due to much higher viscosity and faster hardening $\left(135^{\circ} \mathrm{C}, 700 \mathrm{kPa}\right.$ and $\left.3 \mathrm{~mm} / \mathrm{s}\right)$.

$M_{n} 45000$ showed significant higher mechanical values than $M_{n} 97000$ with the same geometry (Fmax $12.69 \pm 1.48 \mathrm{~N}$ vs. $6.28 \pm 1.60 \mathrm{~N}$ and stiffness $5.72 \pm 0.97 \mathrm{~N} / \mathrm{m}$ vs. $2.40 \pm 0.70 \mathrm{~N} / \mathrm{m}$, respectively). Changing the geometry of $\mathrm{M}_{\mathrm{n}} 97000$ mats led to increased mechanical values (Fmax $8.51 \pm$ $1.96 \mathrm{~N}$ and stiffness $3.15 \pm 0.84$ ).

\section{Conclusion}

Suitable 3D printing parameters could be found for both PCL types, although handling of $M_{n}$ 97000 PCL was more challenging. $M_{n} 97000$ mats showed significantly lower mechanical values than $M_{n} 45000$ mats of the same geometry. Values could be increased by adapting the geometry.

This research was funded by DFG/WE 4262/6-2 as part of the FOR 2180. 